\title{
Correlation between urinary albumin to creatinine ratio and systemic glycocalyx degrada- tion in pediatric sepsis
}

\author{
Rina A.C. Saragih, ${ }^{1}$ Antonius H. Pudjiadi, ${ }^{2}$ Taralan Tambunan, ${ }^{2}$ Hindra I. Satari, ${ }^{2}$ Diana Aulia, ${ }^{3}$ Saptawati \\ Bardosono, ${ }^{4}$ Zakiudin Munasir, ${ }^{2}$ Munar Lubis ${ }^{1}$
}

${ }^{1}$ Department of Child Health, Faculty of Medicine, Universitas Sumatera Utara, Haji Adam Malik Hospital, Medan, Indonesia

${ }^{2}$ Department of Child Health, Faculty of Medicine, Universitas Indonesia, Cipto Mangunkusumo Hospital, Jakarta, Indonesia

${ }^{3}$ Department of Clinical Pathology, Faculty of Medicine, Universitas Indonesia, Cipto Mangunkusumo Hospital, Jakarta, Indonesia

${ }^{4}$ Department of Clinical Nutrition, Faculty of Medicine, Universitas Indonesia, Cipto Mangunkusumo Hospital, Jakarta, Indonesia

\section{ABSTRACT}

Background: Increased capillary permeability in sepsis is associated with several complications and worse outcomes. Glycocalyx degradation, marked by increased serum syndecan-1 levels, alters vascular permeability, which can manifest as albuminuria in the glomerulus. Therefore, elevated urinary albumin to creatinine ratio (ACR) potentially provides an index of systemic glycocalyx degradation. The aim of this study was to analyze the correlation between urinary ACR and serum syndecan- 1 levels.

Methods: A longitudinal prospective study with repeated cross-sectional design was conducted on children with sepsis in pediatric intensive care unit, we evaluated serum syndecan-1 levels and urinary ACR on days 1, 2, 3, and 7. A descriptive study on healthy children was also conducted to determine the reference value of syndecan-1 in children.

Results: 49 subjects with sepsis were recruited. Based on the data of the healthy children group ( $n=30)$, syndecan-1 level of $>90$ th percentile $(41.42 \mathrm{ng} / \mathrm{mL})$ was defined as systemic glycocalyx degradation. The correlation coefficients ( $\mathrm{r}$ ) between urinary ACR and syndecan- 1 levels were $0.32(\mathrm{p}<0.001)$ from all examination days (162 specimens), 0.298 ( $\mathrm{p}=0.038$ ) on day 1 , and 0.469 $(\mathrm{p}=0.002)$ on day 3 . The area under the curve of urinary ACR and systemic glycocalyx degradation was $65.7 \%$ (95\% CI $54.5 \%-$ $77 \%$; $=0.012$ ). Urinary ACR $\geq 157.5 \mathrm{mg} / \mathrm{g}$ was determined as the cut-off point for glycocalyx degradation, with a sensitivity of $77.4 \%$ and a specificity of $48 \%$.

Conclusion: Urinary ACR showed a weak correlation with systemic glycocalyx degradation, indicating that the pathophysiology of elevated urinary ACR in sepsis is not merely related to glycocalyx degradation.

Keywords: glycocalyx degradation, sepsis, syndecan-1, urinary albumin: creatinine ratio

pISSN: 0853-1773 • eISSN: 2252-8083 • https://doi.org/10.13181/mji.v27i3.2156• Med J Indones. 2018;27:194-200

- Received 14 Jul 2017 • Accepted 23 Jun 2018

Corresponding author: Rina A.C. Saragih

rina.amalia.srgh@gmail.com

Copyright @ 2018 Authors. This is an open access article distributed under the terms of the Creative Commons Attribution-NonCommercial 4.0 International License (http://creativecommons.org/licenses/by-nc/4.0/), which permits unrestricted non-commercial use, distribution, and reproduction in any medium, provided the original author and source are properly cited. 
Despite the advanced developments made in the treatment of sepsis, its morbidity and mortality remain high. An epidemiological study in the US reported that the incidence of pediatric severe sepsis is 0.56 cases per 1000 population per year, with a mortality rate of $10.3 \% .{ }^{1}$ Increased capillary permeability in sepsis leads to extravasation of plasma proteins and fluids. Vascular leakage is associated with several complications such as organ edema, hypovolemia, and shock, which are related to worse outcomes and may lead to death. ${ }^{2,3}$ Despite its clinical importance, there is still a lack of a feasible bedside technique for routine quantification of plasma leakage in clinical practice. ${ }^{3}$ Fluid extravasation can be detected by a transcapillary radioactive albumin injection ${ }^{4}$ and a transpulmonary thermodilution; ${ }^{5}$ however, these methods are invasive and relatively expensive.

Glycocalyx disruption is associated with an increased paracellular permeability and an extravasation of albumin or fluid into the interstitial space. ${ }^{6}$ Glycocalyx integrity can be analyzed using the visualization technique or the examination of the glycocalyx degradation marker. ${ }^{3,7}$ Syndecan- 1 is considered to be a valid marker of endothelial glycocalyx integrity. ${ }^{3}$ Superficial luminal endothelium formed by glycocalyx is the earliest component that is affected during sepsis. As a component of glycocalyx, syndecan-1 is stimulated by a mediator of inflammation, which results in its shedding into the blood circulation. ${ }^{8}$ Syndecan-1 levels are not routinely evaluated in the clinical setting, as it is expensive.

Recently, the term "microalbuminuria" is not recommended. ${ }^{9}$ Earlier, microalbuminuria was defined when the urinary albumin excretion per day was 30-300 mg or the urinary albumin to creatinine ratio (ACR) was 30-299 mg/g. Urinary $\mathrm{ACR}>300 \mathrm{mg} / \mathrm{g}$ is clinically defined as proteinuria, whereas urinary ACR $<30 \mathrm{mg} / \mathrm{g}$ is normally found in the healthy population. Although these ranges are also used in the pediatric population, these values are based on research in adults..$^{10}$ Elevated urinary ACR is often detected among critically ill patients and described as a predictor of organ failure, disease severity, and mortality. ${ }^{11,12}$ The mechanism involved in the association between elevated urinary ACR and organ dysfunction has not yet been fully understood. ${ }^{4}$ Glycocalyx disruption leads to an alteration in permeability, and it is clinically manifested as albuminuria in the glomerulus. Wide disruption of glycocalyx is predicted to cause albuminuria and systemic permeability alteration. Therefore, elevated urinary ACR potentially indicates an index of vascular function associated with glycocalyx. ${ }^{13}$ This study was conducted to analyze the correlation between urinary ACR and serum syndecan-1 levels as a marker of endothelial glycocalyx integrity.

\section{METHODS}

This study was approved by the Institutional Review Board of the Universitas Indonesia with the number 64/UN2.F1/ ETIK/2015. A prospective longitudinal study with a cross-sectional design was conducted among septic children in the pediatric intensive care unit (PICU) of Cipto Mangunkusumo Hospital, Jakarta; Haji Adam Malik Hospital, Medan; and Kariadi Hospital, Semarang. The inclusion criteria were children aged 1-35 months, admitted to the PICU, diagnosed with sepsis, and whose parents agreed to participate in the study by signing the informed consent. The diagnostic criteria of sepsis were based on the international pediatric sepsis consensus conference. ${ }^{14}$ Sepsis was defined as a systemic inflammatory response syndrome (SIRS) in the presence of or because of suspected or proven infection. SIRS was defined as the presence of at least two of the following four criteria, one of which must be abnormal temperature or leukocyte count: (1) core temperature of $>38.5^{\circ} \mathrm{C}$ or $<36^{\circ} \mathrm{C},(2)$ tachycardia, defined as a mean heart rate of $\geq 2$ SD above the normal value for age in the absence of external stimulus, chronic drugs, or painful stimuli, or unexplained persistent elevation over a 0.5 - to 4 -h time period OR for children aged $<1$ year; bradycardia, defined as a mean heart rate of $<10^{\text {th }}$ percentile for age in the absence of external vagal stimulus, $\beta$-blocker drugs, or congenital heart disease, or unexplained persistent depression over a 0.5 -h time period, (3) mean respiratory rate $>2 \mathrm{SD}$ above normal for age or mechanical ventilation for an acute process not related to an underlying neuromuscular disease or the receipt of general anesthesia, and (4) leukocyte count elevated or depressed for age (not secondary to chemotherapy-induced leukopenia) or $>10 \%$ of immature neutrophils. 
Infection was defined as a suspected or proven (using positive culture) infection caused by any pathogen or a clinical syndrome associated with a high probability of infection. The evidence of infection included positive findings on the clinical examination, imaging, or laboratory tests (e.g., white blood cells in a normally sterile body fluid, perforated viscous, chest radiograph consistent with pneumonia, petechial or purpuric rash, or severe purpura). Patients with a history of diabetes mellitus, hypertension, or renal disorder and patients with severe allergic reaction were excluded. Syndecan-1 levels and urinary ACR were evaluated on days $1,2,3$, and 7 since the diagnosis of sepsis in the PICU. Disease severity was evaluated using the pediatric logistic organ dysfunction (PELOD) score. Syndecan-1 levels and urinary ACR were evaluated at Prodia Clinical Laboratory Jakarta.

A descriptive study was also conducted on healthy children to determine the reference value for syndecan-1, as we did not find any reference value for syndecan-1 in normal children prior to this study in the literature. We evaluated syndecan-1 level more than $90^{\text {th }}$ percentile as a value that reflects systemic glycocalyx degradation. The inclusion criteria were children aged 1-35 months and defined as healthy based on anamnesis and physical examination performed by a pediatrician. This study also included children who were under preparation for an elective surgery, without a systemic disorder or any other diagnosis besides the indication of the elective surgery. Children with abnormal laboratory results (hemoglobin, hematocrit, leukocyte, thrombocyte, serum glutamic pyruvic transaminase, creatinine, and blood glucose levels and urinalysis) were excluded.

\section{Statistical Analysis}

Mann-Whitney $U$ test was used for analyzing the continuous variables. The correlation between urinary ACR and syndecan-1 levels was analyzed using the Spearman's rank correlation. A receiver operating characteristic (ROC) curve was plotted to statistically analyze urinary ACR as a marker of systemic glycocalyx degradation. Syndecan-1 value more than $90^{\text {th }}$ percentile in healthy children was determined as systemic glycocalyx degradation. Differences considered to be significant when the $p$ value was $<0.05$, and all analyses were performed using Statistical Package for Social Science (SPSS) version 20.0 .

\section{RESULTS}

\section{Study on healthy children}

A total of 30 healthy children, 12 boys and 18 girls, were included in this study, with the median age being $23(2-35)$ months. The median urinary ACR value was 10.5 (3-88) $\mathrm{mg} / \mathrm{g}$, and the mean syndecan-1 level was 27.7 (SD 2.24) ng/mL. Syndecan-1 level $>41.42 \mathrm{ng} /$ $\mathrm{mL}\left(90^{\text {th }}\right.$ percentile) was determined as systemic glycocalyx degradation.

\section{Study on septic children}

A total of 49 septic patients were recruited, consisting of $32(65.3 \%)$ boys and 17 (34.7) girls. Table 1 shows the characteristics of the septic subjects. Syndecan-1 level was increased in the septic group compared to that in the healthy children group [83.40 (10.10-2257.91) ng/mL vs 27.7 (SD 2.24) ng/mL; $\mathrm{p}<0.001$ ]. Syndecan-1 level of $>41.42 \mathrm{ng} / \mathrm{mL}$ was found in $40(81.6 \%)$ subjects on day 1 . The increase in the syndecan- 1 value and the urinary ACR are presented in Table 2. ACR was increased in the septic group compared to that in the healthy group [489.0 (10.0-5446.0) $\mathrm{mg} / \mathrm{g}$ vs 10.5 (3-88) mg/g; $p<0.001]$. A total of 33 (67.3\%) subjects had urinary ACR $>300 \mathrm{mg} / \mathrm{g}$ on day 1 . Figure 1 shows the box plot of urinary ACR and syndecan-1 levels of healthy and septic children.

\section{Correlation between urinary ACR and syndecan-1 Levels}

There were a weak correlation $(\mathrm{r}=0.32$; $\mathrm{p}<0.001$ ) between urinary ACR and syndecan-1 levels for all the 162 paired specimens from all examination days. The correlation analysis carried out on each examination day revealed a weak correlation on day 1 and a moderate correlation on day 3 (Figure 2).

\section{Cut-off point of urinary ACR as a surrogate marker of systemic glycocalyx degradation}

Based on the results obtained for the healthy children group, systemic glycocalyx degradation was defined when the syndecan-1 level was $>41.42 \mathrm{ng} / \mathrm{mL}$. Using the ROC curve, the area under the curve (AUC) of urinary ACR, used as a marker of systemic glycocalyx degradation, was 65.7\% (95\% CI 54.5\%-77\%; $\mathrm{p}=0.012$ )(Figure 3). 
Table 1. Characteristic of septic patients

\begin{tabular}{lc}
\hline Variable & Median (min-max) or $\mathrm{n}(\%)$ \\
\hline Age (months) & $8.0(1.0-32.0)$ \\
PELOD score & $2(0-33)$ \\
$\quad \geq 20$ & $15(30.6)$ \\
$\quad<20$ & $34(69.4)$ \\
Source of infection & \\
Central Nervous System & $14(28.6)$ \\
(CNS) & $22(44.9)$ \\
\multicolumn{1}{c}{ Pulmonary } & $12(24.5)$ \\
$\quad$ Abdominal & $1(2)$ \\
$\quad$ Burn & $83.40(10.10-2257.91)$ \\
\hline
\end{tabular}

PELOD score=pediatric logistic of organ dysfunction

Table 2. Proportion of increased syndecan-1 and urinary albumin to creatinine ratio

\begin{tabular}{|c|c|c|c|c|c|}
\hline \multirow{2}{*}{ Day } & \multirow{2}{*}{$\mathrm{n}$} & \multirow{2}{*}{$\begin{array}{c}\text { Syndecan-1 } \\
>41.42 \mathrm{ng} / \mathrm{mL}, \\
\mathrm{n}(\%)\end{array}$} & \multicolumn{3}{|c|}{ Urinary ACR, n (\%) } \\
\hline & & & $<30$ & $30-300$ & $>300$ \\
\hline 1 & 49 & $40(81.6)$ & $\begin{array}{c}5 \\
(10.2)\end{array}$ & $\begin{array}{c}11 \\
(22.4)\end{array}$ & $\begin{array}{c}33 \\
(67.3)\end{array}$ \\
\hline 2 & 46 & 39 (84.8) & $\begin{array}{c}4 \\
(8.7)\end{array}$ & $\begin{array}{c}13 \\
(28.3)\end{array}$ & $\begin{array}{c}29 \\
(63.0)\end{array}$ \\
\hline 3 & 41 & $34(82.9)$ & $\begin{array}{c}2 \\
(4.9)\end{array}$ & $\begin{array}{c}17 \\
(41.5)\end{array}$ & $\begin{array}{c}22 \\
(53.7)\end{array}$ \\
\hline 7 & 26 & $24(92.3)$ & $\begin{array}{c}2 \\
(7.7)\end{array}$ & $\begin{array}{c}14 \\
(53.8)\end{array}$ & $\begin{array}{c}10 \\
(38.5)\end{array}$ \\
\hline
\end{tabular}

ACR=albumin to creatinine ratio

Urinary ACR $\geq 157.5 \mathrm{mg} / \mathrm{g}$ was determined as the cut-off point for systemic glycocalyx degradation, with a sensitivity of $77.4 \%$, a specificity of $48 \%$, a positive likelihood ratio of 1.49 , a negative likelihood ratio of 0.47 , a positive predictive value of $89 \%$, and a negative predictive value of $28 \%$.

\section{DISCUSSION}

Thereare limited studies on elevated urinary ACR and its reference value in healthy children. . $^{15,16}$ In this study, we found the median urinary ACR as 10.5 (3-88) $\mathrm{mg} / \mathrm{g}$, Urinary ACR was measured among healthy children aged 1-35 months. A study in normal Korean children reported that urinary
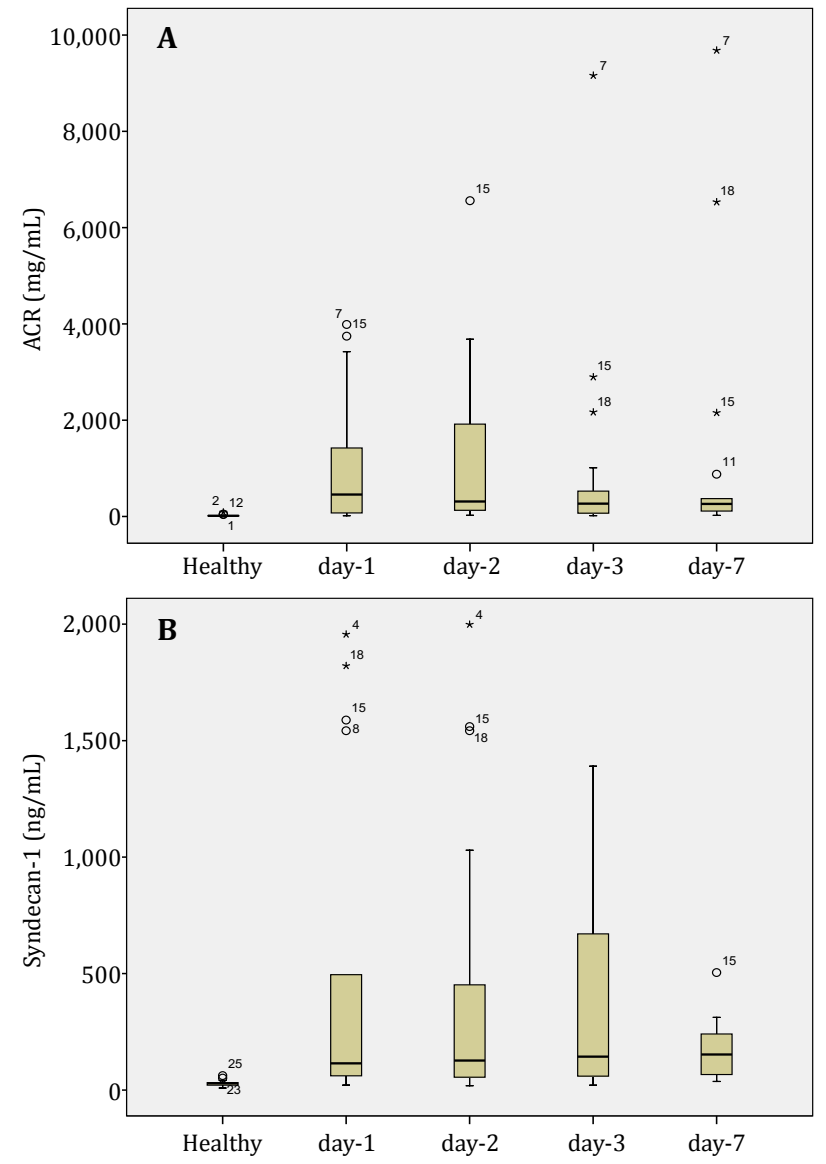

Figure 1. Box plot of urinary ACR (A) and syndecan-1 (B) of healthy and septic children

ACR decreased with age, with the mean values being $22.72(\mathrm{SD}=13.8) \mathrm{mg} / \mathrm{mmol}$ for the age group of $1-12$ months and $16.34(\mathrm{SD}=9.58) \mathrm{mg} / \mathrm{mmol}$ for the age group of 13-28 months. ${ }^{15}$ In our study, we found the urinary ACR as $>30 \mathrm{mg} / \mathrm{g}$ in healthy subjects. This value was similar to that reported by Hurtado et al which demonstrated that elevated urinary ACR can be found in healthy subjects. ${ }^{17}$ Pediatric population is an ideal group for studying urinary ACR because of the relatively minimal multiple morbidities that are commonly found in adults, ${ }^{16}$ such as diabetes and hypertension.

In the present study, the mean syndecan-1 level in healthy children was $27.7 \pm 2.24 \mathrm{ng} /$ mL. Syndecan-1 value more than $90^{\text {th }}$ percentile $(41.42 \mathrm{ng} / \mathrm{mL})$ was determined as systemic glycocalyx degradation. We did not find any reference value for syndecan-1 levels in normal children before this study in the literature. Çekiç et al have reported a mean syndecan-1 value of $34.1(\mathrm{SD}=8.0) \mathrm{ng} / \mathrm{mL}^{18}$ in the healthy adult group. 

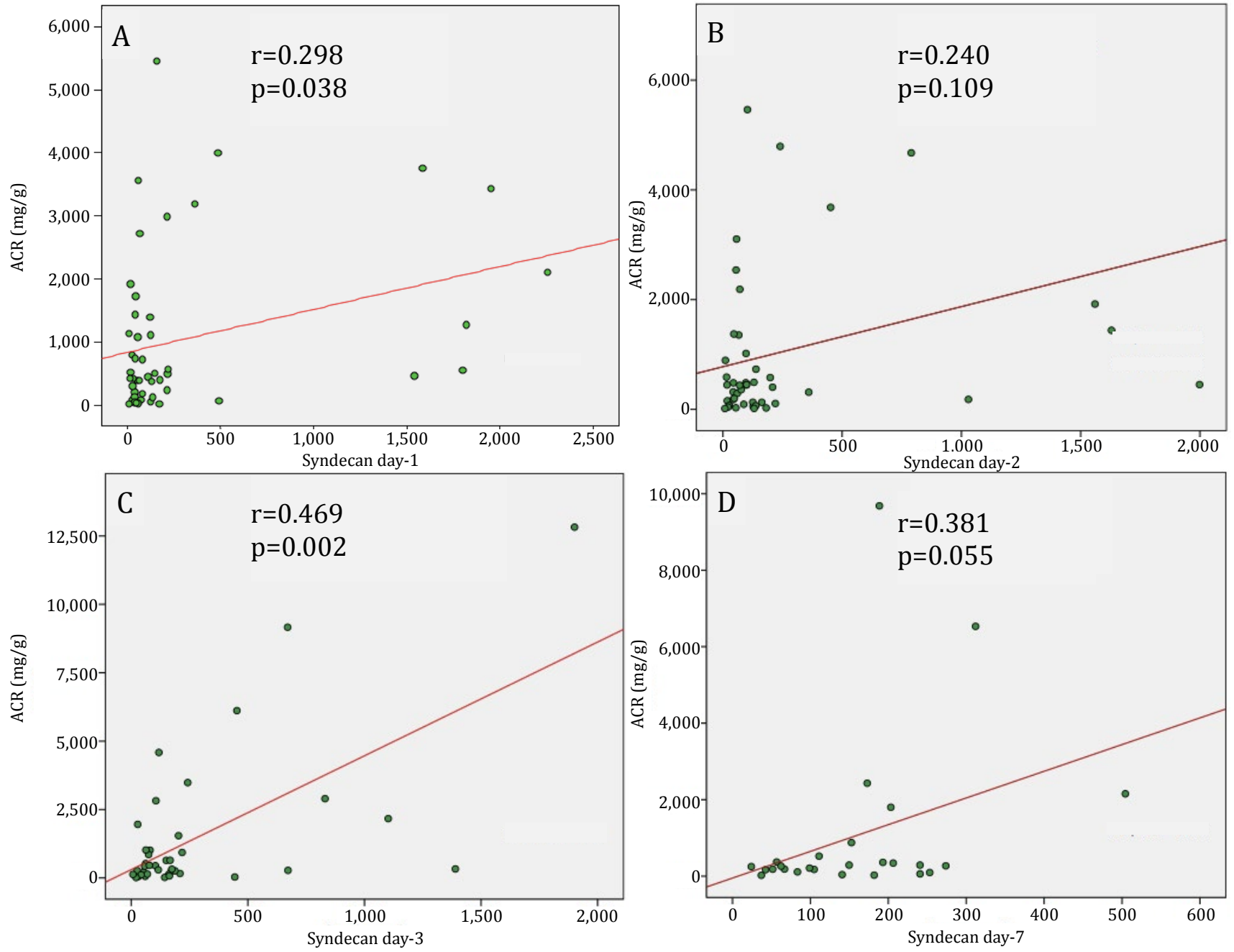

Figure 2. Correlation between urinary ACR and syndecan-1 on days 1 (A) 2 (B), 3 (C), and 7 (D)

Several biomarkers for endothelium have been reported, each with different functions. ${ }^{19}$ In this study, syndecan-1 was used as a marker, which is directly related to the movement of fluid from the intravascular to the extravascular environment. Syndecan-1 is a major component of glycocalyx whose levels are increased in the plasma when glycocalyx shedding occurs. Several studies have reported that infections caused by pathogens may induce syndecan- 1 shedding. ${ }^{20}$ However, studies on syndecan- 1 in septic children are very limited.

This study found a wide range of syndecan-1 levels in the septic group, which could be due to the variety of disease severity. Glycocalyx damage can range from discrete disturbances in the composition of the entire luminal layer to excessive destruction and degradation, with the loss of the entire glycocalyx. ${ }^{21}$ Rehm et $\mathrm{al}^{22}$ also found a wide range of increases in syndecan-1 levels in patients with ischemia undergoing major vascular surgery. Variations in the glycocalyx thickness, basal turnover, degradation rate, and severity are considered to influence the variations in syndecan-1 levels. Furthermore, the glycocalyx component appears to be excreted rapidly by the renal route; therefore, we cannot ensure that the blood sample was collected at the peak time of shedding. ${ }^{22}$

Urinary ACR was found to be increased in the septic group compared to that in the healthy group. A total of $33(67.3 \%)$ subjects had urinary ACR $>300 \mathrm{mg} / \mathrm{g}$, and 11 subjects had urinary ACR $30-300 \mathrm{mg} / \mathrm{g}$ on day 1 . Other studies, using the previous definition of microalbuminuria, 


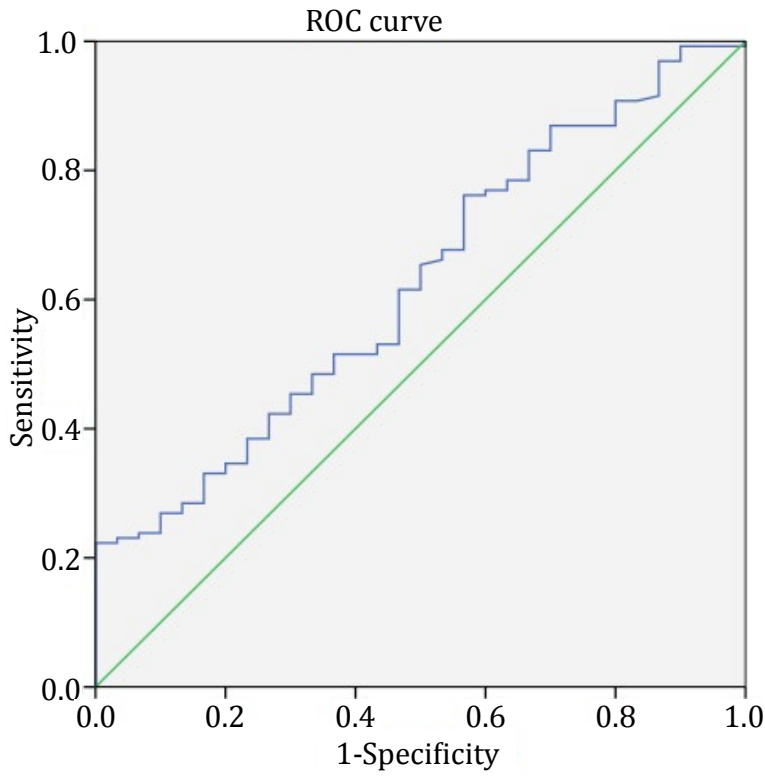

Figure 3. Receiver operating characteristic curve of urinary albumin to creatinine ratio as a marker of systemic glycocalyx degradation

reported the microalbuminuria proportion as $64 \%$ in the pediatric ICU. ${ }^{14}$

Although other study had indicated that increased urinary ACR is a marker of systemic endothelium barrier and permeability disruption caused by sepsis, ${ }^{7}$ this study found a weak correlation between urinary ACR and syndecan-1 levels. Analysis on each examination day also showed a weak correlation on day 1 and a moderate correlation on day 3 . The weak correlation appeared to indicate that the pathophysiology of elevated urinary ACR in sepsis, particularly in the pediatric population, is not merely related to glycocalyx degradation causing altered permeability. Reabsorption of protein by the renal tubules is another factor influencing protein concentration in urine. ${ }^{23}$ A previous study using Pediatric Risk of renal dysfunction, Injury to the kidney, Failure of kidney function, Loss of kidney function, and End-stage Renal Disease (pRIFLE) criteria in the pediatric ICU demonstrated significant improvement of renal function on day 3 of admission, ${ }^{24}$ and another study by Ackan-Arikan et al also reported that renal function showed substantial improvement within $48 \mathrm{~h}$ in $46 \%$ of patients with acute kidney injury. ${ }^{25}$ Improvement in renal tubular function on the third day of admission may be demonstrated using a better correlation between urinary ACR and syndecan-1 levels on day 3 compared to that on day 1; however, we did not examine any marker of tubular function.

In this study, the AUC of urinary ACR as a marker of systemic glycocalyx degradation was $65.7 \%$. Statistically, this AUC showed a weak diagnostic power. However, as there is still a lack of a clinically feasible measurement of plasma leakage in daily practice, particularly related to glycocalyx degradation, this urinary ACR can be useful because it is not invasive, of relatively low cost, and commonly available. It can be used as a surrogate marker to detect the severity of glycocalyx degradation. Therefore, we prioritized the sensitivity in determining the cut-off point of urinary ACR as a marker of systemic glycocalyx degradation in this study. Urinary ACR $\geq 157.5 \mathrm{mg} / \mathrm{g}$ was determined as the cut-off point, with a sensitivity of $77.4 \%$ and a specificity of $48 \%$. This was an interesting finding as the cut-off point, according to the previous definition, was still in the range of the definition of microalbuminuria.

Some limitations still exist in this study. As in daily clinical practice, the disease course and severity on the day of PICU admission were variable, resulting in the wide data range. The decrease in the sample size after day 1, especially on day 7 , caused a limitation in the subgroup analysis per day. Moreover, we also did not measure any marker of renal tubular disruption. Further investigation, including other factors that may influence protein concentration in urine, such as protein reabsorption by the renal tubules, is still needed.

In conclusion, urinary ACR showed a weak correlation with systemic glycocalyx degradation. The weak correlation appeared to indicate that the pathophysiology of elevated urinary ACR in sepsis, particularly in the pediatric population, is not merely related to glycocalyx degradation. Nevertheless, as there is still a lack of a clinically feasible measurement of systemic glycocalyx degradation, this urinary ACR can be considered as a surrogate marker because it is noninvasive, relatively low cost, and commonly available.

\section{Conflict of Interest}

The authors affirm no conflict of interest in this study. 


\section{REFERENCES}

1. Watson RS, Carcillo JA, Linde-Zwirble WT, Clemont G, Lidicker J, Angus DC. The epidemiology of severe sepsis in children in the United States. Am J Respir Crit Care Med. 2003;167(5):695-701.

2. Pawitan JA. Potential agents against plasma leakage. ISRN Pharmacol. 2011;2011:975048.

3. Chappell D, Westphal M, Jacob M. The impact of the glycocalyx on microcirculatory oxygen distribution in critical illness. Curr Opin Anaesthesiol. 2009;22(2):155-62.

4. Ochonidky P, Henning RH, van Dokkum RP, de Zeeuw D. Microalbuminuria and endothelial dysfunction: emerging targets for primary prevention of end-organ damage. J Cardiovasc Pharmacol. 2006;47:S151-62.

5. Bendjelid K, Giraud R, Siegenthaler N, Michard F. Validation of a new transpulmonary thermodilution system to assess global end-diastolic volume and extravascular lung water. Crit Care. 2010;14(6):R209.

6. Chelazzi C, Villa G, Mancinelli P, De Gaudio AR, Adembri C. Glycocalyx and sepsis-induced alterations in vascular permeability. Crit Care. 2015;19(1):26.

7. Donati A, Tibboel D, Ince C. Towards integrative physiological monitoring of the critically ill: from cardiovascular to microcirculatory and cellular function monitoring at the bedside. Crit Care. 2013;17(Suppl1):S5.

8. Johansen ME. Hemostasis and endothelial damage during sepsis. Dan Med J. 2015;62(8):B5135.

9. Kidney Disease: Improving Global Outcomes (KDIGO) CKD Work Group. KDIGO 2012 clinical practice guideline for the evaluation and management of chronic kidney disease. Kidney inter., Suppl. 2013;3:1-150.

10. Singh A, Satchell SC. Microalbuminuria: causes and implications. Pediatr Nephrol. 2011;26(11):1957-65.

11. Bhadade RR, de Souza R, Harde MJ, Sridhar B. Microalbuminuria: a biomarker of sepsis and efficacy of treatment in patients admitted to a medical intensive care unit of a tertiary referral center. J Postgrad Med. 2014;60(2):145-50.

12. Anil AB, Anil M, Yildiz M, Kamit Can F, Bal A, Gokalp G, et al. The importance of microalbuminuria in predicting patient outcome in a PICU. Pediatr Crit Care Med. 2014;15(5):e220-5.
13. Salmon AH, Satchell SC. Endothelial glycocalyx dysfunction in disease: albuminuria and increased microvascular permeability. J Pathol. 2012;226(4):562-74.

14. Goldstein B, Giroir B, Randolph A. International pediatric sepsis consensus conference: definitions for sepsis and organ dysfunction in pediatrics. Pediatr Crit Care Med 2005;6(1):2-8.

15. Kwak BO, Lee ST, Chung S, Kim KS. Microalbuminuria in normal Korean children. Yonsei Med J. 2011;52(3):476-81.

16. Varda NM. Microalbuminuria in pediatric patients with hypertension. Health. 2013;5(4A):40-6.

17. Hurtado A, Cancino R, Figueroa J, Padilla E, Morales C, Ortiz I, et al. Microalbuminuria in healthy adolescents: a comparative study at high altitude and at sea level. Open Urol Nephrol J. 2014;7:82-5.

18. Cekiç C, Kırcı A, Vatansever S, Aslan F, Yilmaz HE, Alper E, et al. Serum syndecan-1 levels and its relationship to disease activity in patients with Crohn's disease. Gastroenterol Res Pract. 2015;2015:850351.

19. Thorburn K, Baines PB. Broken barriers, biomarkers, and blocking antibodies-endothelial activation in sepsis*: no snowflake in an avalanche ever feels responsible. -Voltaire/François-Marie Arouet (16941778). Pediatr Crit Care Med. 2013;14(7): 720-1.

20. Teng YH, Aquino RS, Park PW. Molecular functions of syndecan-1 in disease. Matrix Biol. 2011;31(1):3-16.

21. Ostrowski SR, Haase N, Müller RB, Møller MH, Pott FC, Perner A, et al. Association between biomarkers of endothelial injury and hypocoagulability in patients with severe sepsis: a prospective study. Crit Care. 2015;19(1):191.

22. Rehm M, Bruegger D, Christ F, Conzen P, Thiel M, Jacob M, et al. Shedding of the endothelial glycocalyx in patients undergoing major vascular surgery with global and regional ischemia. Circulation. 2007;116(17):1896-906.

23. D’Amico G, Bazzi C. Pathophysiology of proteinuria. Kidney Int. 2003;63(3):809-25.

24. Saragih RA, Mandei JM, Yuniar I, Dewi R, Pardede SO, Pudjiadi A, et al. Using pRIFLE criteria for acute kidney injury in critically ill children. Paeditr Indones. 2013;53(1):32-6.

25. Ackan-Arikan A, Zappitelli M, Loftis LL, Washburn KK, Jefferson LS, Goldstein SL. Modified RIFLE criteria in critically ill children with acute kidney injury. Kidney Int. 2007; 71(10):1028-35. 